\title{
Rapid crystallization strategy for manganese (II) - L - Tartrate
}

\author{
D. Saravanabharathi ${ }^{\mathrm{b}}$ and S.C.Murugavel ${ }^{\mathrm{a}}$ \\ dsb.chem@psgtech.ac.in, scm.apsc@psgtech.ac.in \\ ${ }^{a}$ Department of Applied Science,PSG College of Technology, Coimbatore - 641 004, \\ India., ${ }^{b}$ Department of Chemistry, PSG College of Technology, Coimbatore - 641 004, \\ India.
}

\begin{abstract}
Aconcise andrapid strategy to crystallize the manganese tartrate is presented. A reactive crystallization strategy used for successful crystallization of manganese (II)-Ltartrate in which the reaction between aqueous solutions of manganese chloride and Ltartaric acid are involved. This paper highlights the advantages concerning overall yield, purity, quickness and environmental benign nature of the crystallization process. We expect that this crystallization strategy can be extended to various metal tartrate crystals. The as-crystallized manganese (II)-L- tartrate was according to new route was characterized by powder X-ray diffraction technique (PXRD) and Fourier transform infrared (FT-IR) spectroscopy. The electrolytic conductivity and water wetting properties have been investigated.
\end{abstract}

Keywords: L-tartrate, PXRD, electrolytic conductivity, spectroscopy.

\section{Introduction}

Metal tartrate compounds have received a lot of attention in science and technology for a wide range of applications.In general, metal tartrate compounds are formed by thecoordination of alkali or alkaline or transition metal ions with tartaric acids. Metal tartrate crystals have a range of remarkable physical properties, including dielectric, ferroelectric, piezoelectric and other important features [1].Metal tartrates are used in transducers, linear and non-linear mechanical/optical devices, and in the construction of crystal oscillators and resonators because of their remarkable properties [2].

Moreover, metal tartrate compounds are also used in medical, pharmaceutical and industrial fields. Injections of sodium-chromium tartrate compounds, for example, improve the susceptibility of transplanted sarcoma to the effects of X-rays; ferrous tartrate compounds reduce anaemia in animals; tanning action to tan skin; and catalyst in champagne production. [3].

Several authors have reported crystallization and characterization of various metallic tartrate crystals [4-7]. The majority of metal tartrate compounds are insoluble in water and disintegrate prior to melting.Therefore, pure single crystals of these type of compounds difficult to obtain in slow evaporation or melt growth technique. Hence, it is mostly grown using gel phase technique [8]. However, gel phase crystallization is not an appropriate 
method for crystallizing metal tartrate compounds due to maintaining the $\mathrm{pH}$ of the gel medium, contamination of product and it require longer times ranging from days to weeks for the completion of process[ 9]. As a result of this, it is essential to develop alternate method of crystallizations.

It is to be observed that, the literature on the manganese tartrate based crystals, with respect to its modified reactive crystallization strategy remain un-attempted.As a result, the current work proposes an approach for the fast crystallisation of pure manganese (II)-Ltartrate in a concise, environmentally acceptable manner.

\section{Experimental}

\subsection{Materials and Methods}

Manganese(II) chloride tetrahydrate $\left(\mathrm{MnCl}_{2} .4 \mathrm{H}_{2} \mathrm{O}\right)$, L-tartaric acid $\left(\mathrm{C}_{4} \mathrm{H}_{6} \mathrm{O}_{6}\right)$, and anhydrous sodium carbonate $\left(\mathrm{Na}_{2} \mathrm{CO}_{3}\right)$ were employed in this study. The chemicals were used without any further purification and the solvent doubly distilled water was used throughout the investigation.

\subsection{Synthesis of manganese (II)-L- tartrate}

Manganese (II) - L-tartrate was crystallized by reactive crystallization method which involves plain mixing of aqueous solutions of manganese(II) chloride with L-tartaric acid. In a $500 \mathrm{~mL}$ beaker, L-tartaric acid $(20 \mathrm{mmol}, 3 \mathrm{~g}$ ) was dissolved in $200 \mathrm{~mL}$ double distilled water and then treated with $2.06 \mathrm{~g}$ solid sodium carbonate, which was agitated well until no carbon dioxide was released.A manganese (II) chloride tetrahydrate solution was made separately and added to the aforesaid aqueous disodium tartrate solution [20 mmol, 4.04g in $100 \mathrm{~mL}$ of double distilled water].The solution were very lightly swirled to ensure thorough mixing of the reactants and then left undisturbed.Bright pink single crystals developed at the base of the beaker within 24 hours, and the crystallization process completed within 24-48 hours.After being washed with abundant amounts of distilled water,filtered, and dried in the open air, the light pink colour crystals were obtained (Yield: $3 \mathrm{~g}$ ), and the single crystals formed are shown in Figure 2.1.

The chemical reaction takes place in the synthesis is represented in the following scheme 2.1 .

$$
\begin{array}{cccc}
\mathrm{C}_{4} \mathrm{H}_{6} \mathrm{O}_{6}(\mathrm{aq})+\mathrm{Na}_{2} \mathrm{CO}_{3}(\mathrm{aq}) \stackrel{-\mathrm{CO}_{2} \uparrow}{\longrightarrow} & \mathrm{Na}_{2} \mathrm{C}_{4} \mathrm{H}_{4} \mathrm{O}_{6}(\mathrm{aq}) \stackrel{+\mathrm{MnCl}_{2}}{-2 \mathrm{NaCl}} \rightarrow \mathrm{MnC}_{4} \mathrm{H}_{4} \mathrm{O}_{6}(\mathrm{~s}) \\
\text { L-tartaric acid } & \text { Sodium carbonate } & \begin{array}{c}
\text { Disodium tartrate } \\
\text { complex }
\end{array} & \begin{array}{c}
\text { Manganese(II)-L- tartrate } \\
\text { complex }
\end{array}
\end{array}
$$

Scheme 2.1: Synthesis of manganese(II) tartrate complex 


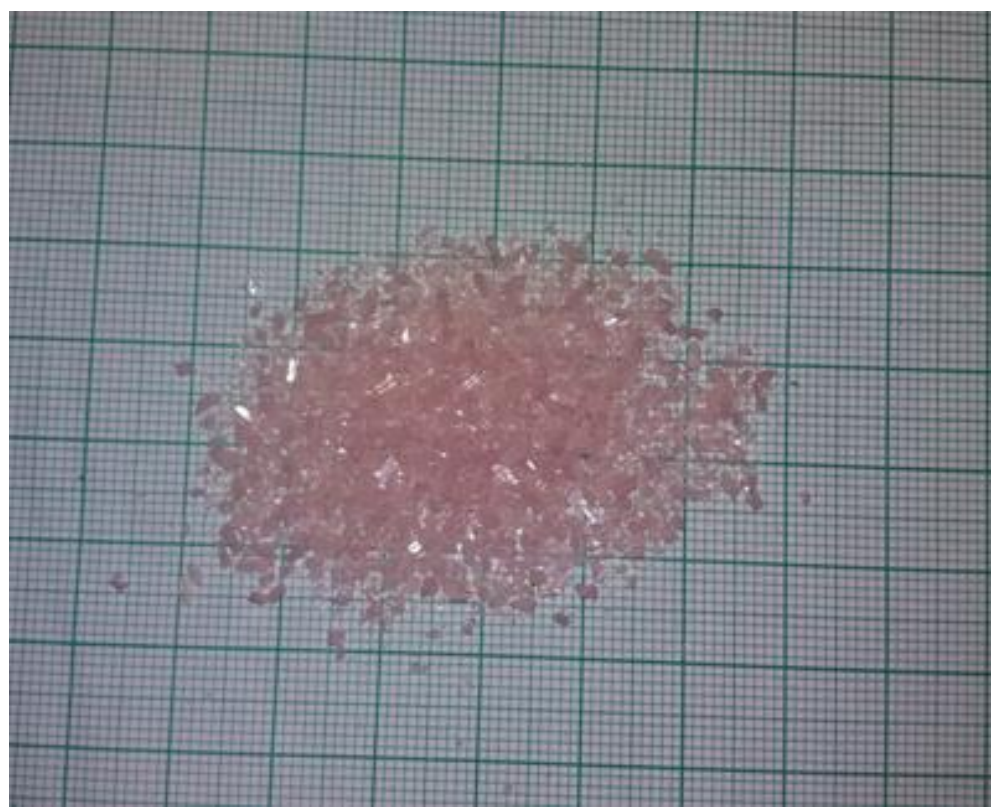

Figure 2.1: Single crystals of manganese(II)-L-tartrate crystallized at room temperature

The synthesized manganese(II)-L- tartrate compound was characterized as follows:

In order to obtain a powder XRD spectrum, the fine powdered manganese-L- tartrate sample was mounted on a Perspex plate using vacuum grease as a binder. The PXRD experiments were performed on Shimadzu Lab Model 6000 XRD instrument with CuKa radiation $(\lambda=1.54060)$ was used.

In order to record FTIR spectrum, a $\mathrm{KBr}$ pellet technique was used, wherein, 5-10 $\mathrm{mg}$ of manganese tartrate sample was thoroughly grounded with $100 \mathrm{mg}$ of solid $\mathrm{KBr}$. The resulting solid mixture was compressed by giving an appropriate pressure to form a thin transparent pellet. The FTIR spectral analysis of powdered and pelletized sample was recorded ranging from $400-4000 \mathrm{~cm}^{-1}$. TheFTIR measurements of the sample were recorded using Shimadzu 8400S FT-IR spectrophotometer.

For solubility determination, powdered sample were added into the water at room temperature $\left(31^{\circ} \mathrm{C}\right)$ and electrolytic conductivity of the solution was measured using direct reading conductivity meter (ELICO CM 180, INDIA). Conductivity water used for measurement obtained from double distillation unit with a specific conductivity of $6 \times 10^{-5} \mathrm{~S}$ $\mathrm{cm}^{-1}$. The conductivity cell was calibrated using $0.1 \mathrm{~N} \mathrm{KCl}$ solution. For conductivity measurement, $100 \mathrm{~mL}$ glass beaker is filled with $50 \mathrm{~mL}$ of double distilled water. The synthesized manganese(II)-L- tartrate compound was added in small amounts at successive stages. The addition of the compound and stirring were continued till a small amount remains insoluble, which confirmed the saturation condition. The saturated solution was then warmed and cooled to room temperature, and the solution's conductivity was calculated. The solubility of synthesized complex were determined using the equation $\Lambda_{m}^{\circ}=1000 \mathrm{~K} / \mathrm{M}$ where, $\Lambda_{m}^{\circ}, \mathrm{K}$ and $\mathrm{M}$ are respectively, the molar conductivity of the solution $\left(\mathrm{S}_{\mathrm{cm}} \mathrm{cm}^{2} \cdot \mathrm{mol}^{-1}\right)$, specific conductance $\left({\mathrm{S} . \mathrm{cm}^{-1}}^{-1}\right.$, and molarity or solubility (g/litre) of the manganese tartrate compound in solution, respectively .The $\Lambda_{m}^{\circ}$, of the manganese tartrate is $226.2 \mathrm{~S} \mathrm{~cm}^{2} \mathrm{~mol}^{-1}$, which are calculated by applying Kohlraush's law of independent migration of ions. 
The powdered samples were pressed into a pellet with a diameter of $1.3 \mathrm{~cm}$ and a thickness of $0.30 \mathrm{~cm}$ at a pressure of $100 \mathrm{~kg} \mathrm{~cm}^{-2}$ for contact angle measurements. The DMs200 (Kyowa, Japan) standard goniometer is used to measure the contact angle of a sessile drop of water on the top of a solid manganese tartrate sample.

\section{Results and Discussion}

\subsection{Syntheses features}

For the synthesis of manganese (II) - L-tartrate in this approach, fully water-soluble reactants were utilised.As a result, the strategy used in this study can be termed a simple and one-pot method.It's also worth noting that, the quickness of crystallization of the current approach and the crystalline products emerged from the mother solution within 24-48 hours.It could be owing to the reactants stoichiometry and its cleanliness (Figure 3.1). On the other hand, the previously documented methods involving the slow evaporation of solvent or the gel medium procedures are usually known form precipitate rather than crystals [10], contamination of the product by other impurities in the gel medium, as well as a significantly longer duration for the completion of the process [11].
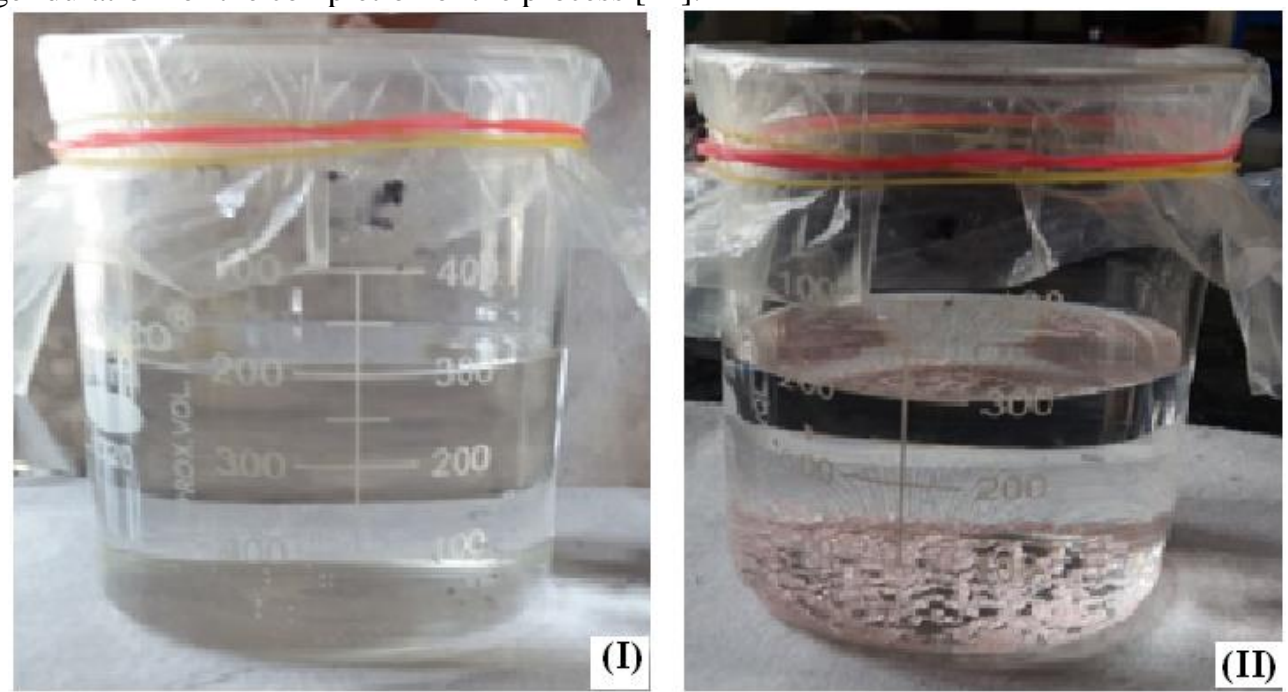

Figure 3.1: The photograph showing the settling of manganese (II)-L-tartrate crystals at the bottom of the reaction beaker at different time intervals; (I) Immediately upon mixing, (II) after $\sim 24$ hours.

\subsection{Powder XRD analysis}

$\mathrm{X}$-ray diffraction is a powerful techniques used for studying phase purity and crystalline nature of the material. Moreover, this technique also provides detailed information of the crystal structures, grain size, orientations and chemical compositions of materials.

The PXRD profile of the synthesized compound, in its powered form, is shown in the Figure 3.2 


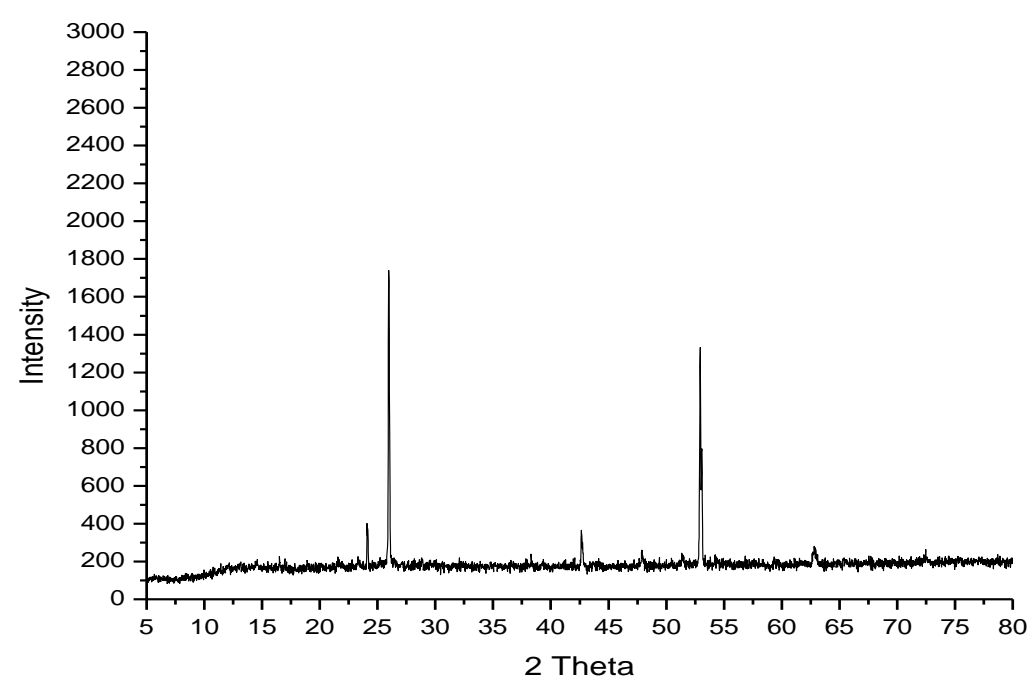

Figure 3.2: PXRD pattern of manganese(II)-L- tartrate complex

It exhibits sharp and high intense diffraction peaks, indicating that the synthesized complex is good crystalline nature.

\subsection{FT-IR analysis}

The FT-IR spectrum was taken in order to understand structural features of the synthesized compound. The FT- IR spectrum of the manganese tartrate shown in the Figure 3.3

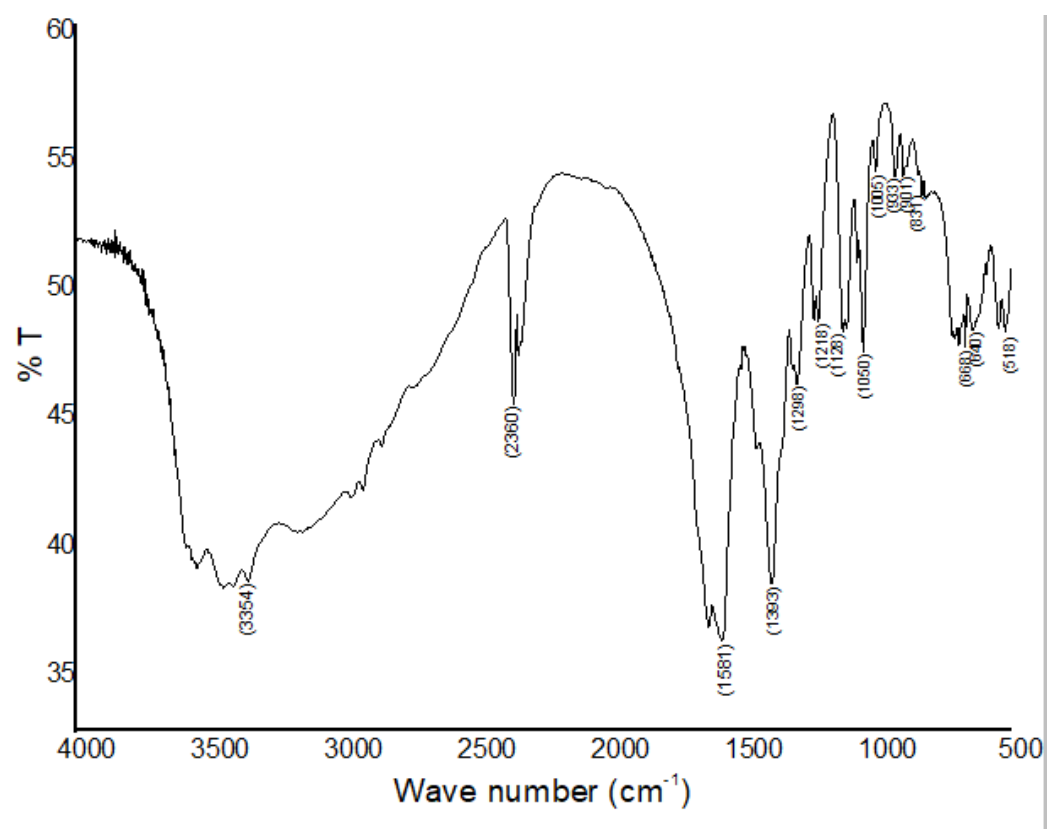

Figure 3.3: FT-IR spectrum of manganese(II)-L- tartrate crystals 
The FT-IR profile of the synthesized compound is very similar to those of the other metal-tartrates described in the literature [12]. Table 4.1 shows the key IR spectral bands and their assignments. The absorption peaks at $3300-3600 \mathrm{~cm}^{-1}$ due to the water of crystallization[4] associated with the crystals, as well as the absence of any peak around $1700 \mathrm{~cm}^{-1}$, offer evidence for the deprotonated nature of carboxylic groups. The IR spectrum reveals that the manganese (II)-L- tartrate crystals obtained in the modified method yield a well-defined crystal with water of crystallization.

Table 3.1: The observed absorption bands $\left(\mathrm{cm}^{-1}\right)$ and their respective assignments of manganese(II)-L-tartrate crystals

\begin{tabular}{|l|l|l|l|}
\hline $\begin{array}{l}\text { Characteristic } \\
\text { absorption }\left(\mathbf{c m}^{-\mathbf{1}}\right)\end{array}$ & Intensity & $\begin{array}{l}\text { Functional group } \\
\text { assignments }\end{array}$ & Types of vibration \\
\hline 3354 & Strong, Broad & $-\mathrm{OH}$ & Stretch \\
\hline 2360 & Strong, Sharp & $\mathrm{CO}_{2}$ & Asymmetric stretch \\
\hline 1581 & Strong & $-\mathrm{C}=\mathrm{O}$ & $\begin{array}{l}\text { Symmetrical and } \\
\text { asymmetrical stretch }\end{array}$ \\
\hline 1393 & Strong, Sharp & $-\mathrm{O}-\mathrm{H}$ & In plane bend \\
\hline 1298 & Weak & $-\mathrm{C}-\mathrm{O}$ & Stretch \\
\hline 1218 & & & Stretch \\
\hline 1128 & Medium & $\mathrm{C}-\mathrm{H}$ & Stretch \\
\hline 1050 & Weak & $\mathrm{C}-\mathrm{OH}$ & Stretch \\
\hline 1005 & Weak & $\mathrm{C}-\mathrm{C}$ & Out of plane bend \\
\hline 933 & Weak & $-\mathrm{O}-\mathrm{H}$ & Rock \\
\hline 901 & Medium & $\mathrm{H}_{2} \mathrm{O}$ & Scissors \\
\hline 831 & Medium & $\mathrm{CO}{ }_{2}$ & Stretch \\
\hline 668 & Medium & $\mathrm{Mn}-\mathrm{O}$ & \\
\hline 640 & & & \\
\hline 518 & & &
\end{tabular}

\subsection{Electrolytic conductivity measurement}

The conductivity value of manganese (II) - L-tartrate was measured by dissolving in water at $31^{\circ} \mathrm{C}$. The conductance values of the manganese (II) $-\mathrm{L}$-tartrate in water were given in Table 4.2.

Table 3.2: Conductance data for manganese (II)-L- tartrate compound with cell constant 1.00.

\begin{tabular}{|c|c|c|c|c|}
\hline \multicolumn{5}{|c|}{ 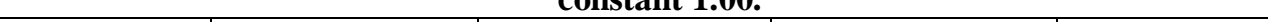 } \\
\hline $\begin{array}{l}\text { Number of } \\
\text { attempts } \\
\text { made }\end{array}$ & $\begin{array}{c}\text { Conductance } \\
\text { (S) }\end{array}$ & $\begin{array}{c}\text { Specific } \\
\text { conductance } \\
\left(\mathrm{S} . \mathrm{cm}^{-1}\right)\end{array}$ & $\begin{array}{c}\text { Molar } \\
\text { conductance } \\
\left(\mathrm{S} . \mathrm{cm}^{2} \cdot \mathrm{mol}^{-1}\right)\end{array}$ & $\begin{array}{l}\text { Solubility } \\
\text { (g/L) }\end{array}$ \\
\hline Trial 1 & $3.1 \times 10^{-5}$ & $3.1 \times 10^{-5}$ & $1.37 \times 10^{-3}$ & 0.278 \\
\hline Trial 2 & $3.0 \times 10^{-5}$ & $3.0 \times 10^{-5}$ & $1.31 \times 10^{-3}$ & 0.263 \\
\hline Trial 3 & $2.5 \times 10^{-5}$ & $2.5 \times 10^{-5}$ & $1.10 \times 10^{-3}$ & 0.224 \\
\hline
\end{tabular}


From the conductivity measurement, it is observed that molar conductance and solubility of the synthesized compound is very low. It indicates that, the non-electrolyte and sparingly soluble nature of the manganese (II)- L-tartrate.

\subsection{Contact angle measurement}

Water contact angle measurements of manganese (II) $-\mathrm{L}-$ tartrate were done at room temperature. Two distinct specimens were prepared and evaluated in order to obtaintrustworthy results. The contact angle of the each specimen was measured at two different regions. Figure 3.4 (a \& b) show the water contact measurement images of two different specimens of manganese tartrate. The average value is taken as the contact angle of manganese (II) $-\mathrm{L}$-tartrate sample. Therefore, the observed average water contact angle of the manganese tartrate are $20.3^{\circ}$. The result revealed that, the highly hydrophilic behaviour of manganese tartrate. The presence of lattice water molecules and hydroxyl groups in their structure is responsible for its high hydrophilicity.

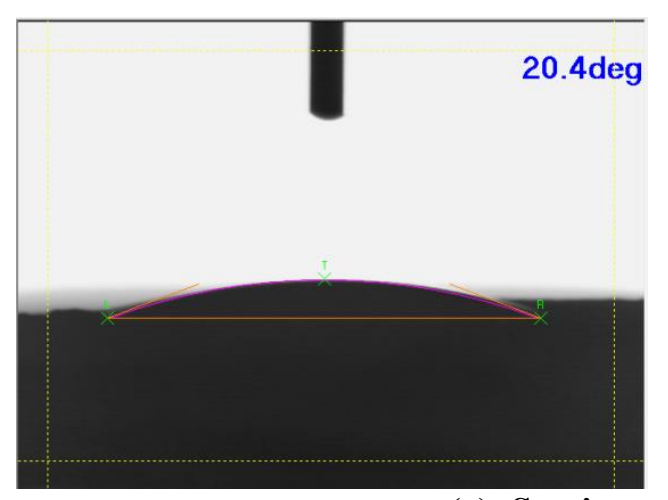

(a) Specimen 1

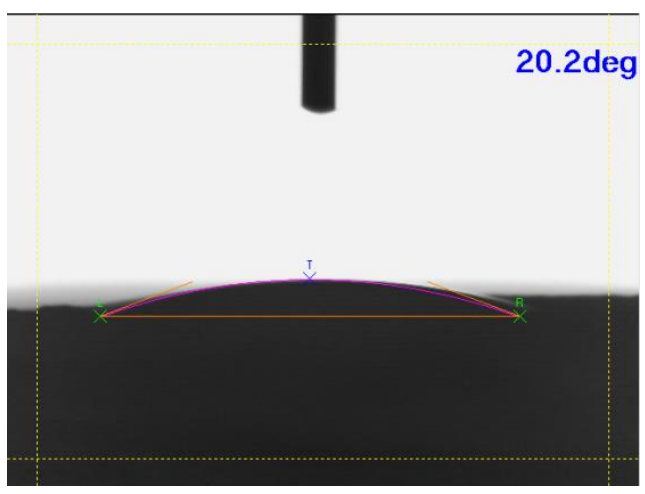

(b) Specimen 2

Figure 3.4: Measurement of water contact angles of (a) Specimen 1 and (b) Specimen 2 whose actual contact value given in the inset.

\section{Conclusion}

In conclusion, we crystallized a manganese (II) - L-tartrate from manganese (II) chloride and L-tartaric acid using reactive crystallization method which possess the following unique features:

i) More environment friendly i.e. crystallization takes place at ambient temperature and pressure without evaporation of the solvent.

ii) Crystallization takes place very quickly.

iii) Simple and easy method.

iv) It is suitable for those materials that undergo decomposition at high temperature and pressure.

v) The use of low temperature for the crystallization avoids thermal shock during growth of the crystal or on removal from the apparatus.

vi) Structural imperfections are relatively low.

The crystals are observed to be transparent and light pink colour. The crystallinity of the material is shown by powder X-ray diffraction examination. The existence of various 
functional groups and crystallisation water was confirmed by FT-IR analysis. The molar conductivity and solubility values confirm the non-electrolytic and sparingly soluble nature of the crystals. Water contact angle measurement of manganese(II))-L- tartrate is $20.3^{\circ}$ reveals the highly hydrophilic nature of the crystals. The properties wettability and sparingly soluble nature of manganese (II) - L-tartrate signify the various technological applications of the materials.

\section{References}

[1] Suresh, S., S.R. Devi, B.M. Sornamurthy, M. Arivanandhan and R.M. Kumar, 2019. Growth, structural and optical studies of a novel nonlinear optical material: p-Toluidinium L-Tartrate. Optik, 185: 651-656.

[2] Brown, W.H., 2016. Tartaric acid. Britannica. https://www.britannica.com/science/tartaric-acid.

[3] Growth and characterization of gel grown pure and mixed iron-Manganese Levo-tartrate crystals JOSHI et al. Department of Physics, Saurashtra University, Rajkot 360 005, India.

[4] A.C.Yanes, T.Lopez, J.Stockel, J.F.Peraza, M.E.Torres 1996.Department of basic and Experimental Physics. 'Characterization and Thermal and Electromagnetic behaviour of Manganese Tartrate crystals grown by Silica gel Technique'.

[5] Ghosh, A.K., E.S. Koltun and G. Bilcer, 2001. Tartaric acid and tartrates in the synthesis of bioactive molecules. Synthesis, 9: 1281-1301.

[6] David, G.S., S. Revathi and M.B.J. Raj, 2014. Structural and optical characterisation of zinc doped potassium hydrogen tartrate crystal for antibacterial analysis. Scholars J. Eng. Technol., 2: 795802.

[7] Ramakrishnan, V \&Maroor, JM 1988, 'IR and Raman studies of gel grown manganese tartrate', Infrared physics, vol. 28, no. 4, pp. 201-204.

[8] Selasteen, F.D. and Raj, S.A.C., 2021. A study on non-linear optical properties of Copper Sodium Tartrate single crystal for non-linear optical applications. Indian Journal of Science and Technology, 14(2), pp.101-112.

[9] Patel, A.R. and Rao, A.V., 1982. Crystal growth in gel media. Bulletin of Materials Science, 4(5), pp.527-548.

[10] H. Soylu* .Department of Physics, Hacettepe University, Ankara, Turkey 1985.

[11] Jethva, H., 2017. Gel growth: a brief review. Mechanics, Materials Science \& Engineering MMSE Journal. Open Access, 9.

[12] Gandhimathi, R., Krishnan, C.M. and Selvarajan, P., 2015. Growth and characterization of manganese sulpho tartrate (MST) - A semiorganic NLO crystal. Optik, 126(21), pp.2925-2929. 